60-61| 2018

La culture dans l'enseignement du français langue étrangère: conceptions théoriques, programmes et manuels auX $\mathrm{XIX}^{\mathrm{e}}$ et $\mathrm{XX}^{\mathrm{e}}$ siècles

\title{
Vladislav Rjéoutski and Willem Frijhoff (edited by). Language Choice in Enlightenment Europe. Education, Sociability, and Governance.
}

Amsterdam : Amsterdam University Press, «Languages and Culture in History », 2018, 234 pages. ISBN : 9789462984714 ; e-ISBN : 978904853 5507 (pdf).

Henri Besse

\section{OpenEdition Journals}

\section{Édition électronique}

URL : https://journals.openedition.org/dhfles/5805

DOI : $10.4000 /$ dhfles.5805

ISSN : 2221-4038

Éditeur

Société Internationale pour l'Histoire du Français Langue Étrangère ou Seconde

Édition imprimée

Date de publication : 1 janvier 2018

ISSN : 0992-7654

Référence électronique

Henri Besse, « Vladislav Rjéoutski and Willem Frijhoff (edited by). Language Choice in Enlightenment Europe. Education, Sociability, and Governance. », Documents pour I'histoire du français langue étrangère ou seconde [En ligne], 60-61 | 2018, mis en ligne le 31 mai 2019, consulté le 31 mars 2023. URL : http:// journals.openedition.org/dhfles/5805 ; DOI : https://doi.org/10.4000/dhfles.5805 


\section{Vladislav Rjéoutski and Willem} Frijhoff (edited by). Language Choice in Enlightenment Europe. Education, Sociability, and Governance.

Amsterdam : Amsterdam University Press, «Languages and Culture in History », 2018, 234 pages. ISBN : 9789462984714 ; e-ISBN : 978904853 5507 (pdf).

\section{Henri Besse}

1 Cet ouvrage, le sixième de la jeune collection Languages and Culture in History dirigée par Willem Frijhoff et Karène Sanchez-Summerer, regroupe huit contributions, précédées d'une introduction synthétique et suivies de deux index, un relatif aux langues ou dialectes (Languages) qui y sont nommés, l'autre aux personnages, auteurs et lieux cités (Persons and places). Trois de ses contributeurs sont bien connus des lecteurs de Documents: Willem Frijhoff et Vladislav Rjéoutski qui, outre leur commune introduction, $\mathrm{y}$ ont chacun rédigé une contribution; Madeleine van StrienChardonneau qui nous a fait connaître, en décembre 1999 à Utrecht, Isabelle de Charrière née Belle de Zuylen. Cinq leur sont moins connus: Andrea Bruschi de l'université de Vérone; Olga Khavanova de l'Académie des sciences de Moscou; Ivana Horbec et Maja Matasovic de l'Institut croate d'histoire de Zagreb; Michael Rocher diplômé des universités de Potsdam et de Halle ; Ekaterina Kislova de l'université d'État de Moscou'.

Quant au « choix des langues dans l'Europe des Lumières » qui titre cet ouvrage, il y est question du français (25 références dans son index Languages) mais bien d'autres langues, tant savantes (latin classique ou non, grec ancien ou byzantin, slavon, hébreu) que vulgaires (italien, allemand, russe, anglais...), y ont quasi autant de références, sans compter nombre de dialectes moins référencés.

3 Voltaire, dans son Histoire de la guerre de 1741, réduisait ainsi ce multilinguisme européen : « De douze langues qu'on parle en Europe, il faut bien qu'on en choisisse une 
qui soit commune. » Laquelle était évidemment, aux yeux de l'historiographe du roi de France qu'était alors Voltaire, le français. Un des mérites de cet ouvrage est de décrédibiliser par les faits la synecdoque («la partie pour le tout») selon laquelle, de Dominique Bouhours à Marc Fumaroli, «l'Europe parlait français » aux XVII ${ }^{\mathrm{e}}$ et XVIII siècles. Une Europe dont le nombre de parlants français n'allait guère au-delà - $\mathrm{y}$ compris sans doute en France, si l'on s'en tient à la pratique effective du «bon usage » selon Vaugelas - d'un sur vingt (cf. Frijhoff, pour les Pays-Bas, p. 50).

Un des autres mérites de ce volume est que le « choix des langues » n'y est pas raisonné à partir d'une supposée "universalité » du français, mais en fonction de ses emplois attestés dans les fonds d'archives du temps. On pourrait dire qu'on y est plus proche de Johann Christoph Schwab que d'Antoine de Rivarol au concours de Berlin en 1783-1784. Nulle allusion à cette translatio imperii - à la manière dont l'imperium de Troie fut transféré par Énée à celui de Rome - qu'évoque Rivarol au début de son essai, nul recours à cette "clarté française » qui a tant servi à justifier la précellence héréditaire $\mathrm{du}$ français sur les autres langues d'Europe, qu'elles soient savantes ou vulgaires. Une «clarté » qui, tout comme parfois celle de Voltaire, relève plus du convenu que du réfléchi. En termes actuels, la réflexion n'y est plus autocentrée sur la France et son rayonnement en Europe, mais multicentrée selon le passé (pas seulement académique) de ses contributeurs, selon le lieu et la langue à partir desquels ils ont œuvré et selon le point de vue qu'ils ont adopté.

5 Soulignons la qualité du travail éditorial des deux coordinateurs de cet ouvrage. Il paraît sous la couverture en couleurs fortement cartonnée caractéristique de la collection Languages and Culture in History. Celle-ci reproduit une carte du XVIII ${ }^{e}$ siècle où sont calligraphiés les premiers mots du «Pater noster (Our Father) » dans différentes langues ou dialectes européens. Que, dans l'intitulé de cette collection, Languages soit au pluriel et Culture au singulier ne peut manquer d'interroger les enseignants et didacticiens des langues étrangères ou secondes, accoutumés à penser qu'à une langue correspond une culture plus ou moins spécifique (cf. des locutions telles langue-culture ou didactologie des langues et des cultures).

6 Tout dans cet ouvrage est en anglais ou traduit dans cette langue (cf. ci-dessus Pater noster $=$ Our Father), non seulement ses huit contributions et son introduction, mais aussi les citations et les titres des bibliographies (à quelques exceptions près) donnés dans une autre langue que l'anglais. Deux contributions (celle de Madeleine van StrienChardonneau et celle de Michael Rocher) y sont dites traduites de leur langue originale (le français et l'allemand), certaines y ont été écrites en anglais par des non natifs de cette langue mais la maîtrisant bien, d'autres enfin ont été traduites par des non natifs compétents en anglais avant être relues par un anglophone natif. Il en résulte, au sentiment du lecteur non natif que je suis, un anglais qui n'a certes pas la vivacité idiomatique et la souplesse grammaticale inhérente aux textes écrits par des natifs pour des natifs, et encore moins cette intertextualité native qui donne souvent au lecteur non natif la sensation d'entrer en terra incognita. Mais c'est un anglais qui a l'incontestable avantage d'être aisément lisible pour qui n'est pas nativement anglophone.

7 Peut-être d'ailleurs en allait-il de même pour le néo-latin (le terme apparaît dans l'index Languages) tel qu'il était pratiqué au XVIII esiècle par les clercs et les laïcs européens quelque peu éduqués ou cultivés. À une différence près toutefois : ces latinistes avaient tous - rares étaient les femmes à le maîtriser - appris le latin à partir d'à peu près le 
même corpus de textes littéraires et/ou religieux, ce qui leur donnait une culture intertextuelle lettrée plus ou moins commune, dont notre actuel global English ou globish est dépourvu.

8 Frijhoff (p. 51) parle du global French for cosmopolitans dans les Pays-Bas du XVIII ${ }^{\mathrm{e}}$ siècle. Faut-il l'assimiler à cette lingua franca que fut séculairement le latin dans une Europe réduite aux seuls éduqués ou cultivés, ou bien à cette lingua franca, pratiquée tout aussi "universellement» dans les ports de la Méditerranée par les non éduqués, qui empruntait aux divers vernaculaires pratiqués sur ses rives? De quelle intertextualité partagée, cosmopolite si l'on préfère, relevait ce global French? Autant de questions qu'un non natif un peu anglophone peut se poser à la lecture du globish de cet ouvrage, mais qui n'enlèvent rien à la qualité de ses contributions et au soin avec lequel il a été édité.

Chacune de ses huit contributions est suivie d'une bibliographie distinguant les sources non publiées (Unpublished sources, autrement dit des archives inédites) des sources publiées (Published sources and bibliography), certaines de ces huit bibliographies couvrant jusqu'à sept à huit pages en petits caractères. Sources qui sont dûment rappelées, en abrégé et presque toujours traduites en anglais, dans les notes de bas de pages, ce qui évite au lecteur d'avoir à se reporter à la bibliographie finale de chaque contribution. Bibliographie suivie d'une courte notice biographique relative au passé académique de son auteur. Bref, un ouvrage destiné d'abord à des publics universitaires, anglophones de naissance ou non, qui y retrouvent aisément toutes les références nécessaires à leur travail.

Plutôt que de résumer l'une après l'autre ces huit contributions - Rjéoutski et Frijhoff s'y adonnent dans leur introduction (pp. 7-12) et les abstracts précédant chacune d'entre elles y suffisent -, nous regrouperons six d'entre elles deux par deux, parce qu'elles œuvrent à peu près sur le même terrain géographique et sur à peu près les mêmes langues, mais à partir de points de vue différents, ce qui stimule l'intérêt du lecteur en équilibrant les analyses.

11 D'abord celles de Frijhoff et de Van Strien-Chardonneau qui portent surtout, mais pas exclusivement, sur les usages tant parlés qu'écrits du français dans les Pays-Bas des $\mathrm{XVII}^{\mathrm{e}} \mathrm{XVIII}^{\mathrm{e}}$ siècles, Frijhoff adoptant une large focale - il y synthétise magistralement nombre de ses écrits antérieurs en néerlandais, français et anglais -, Van StrienChardonneau une focale plus étroite, celle des archives relatives à trois générations d'une famille patricienne hollandaise, celle des van Hogendorp. Même si Frijhoff accorde plus d'attention aux facteurs sociaux, religieux, commerciaux ou professionnels que Van Strien-Chardonneau, davantage sensible aux facteurs de genre, d'intimité partagée ou d'option politique, l'un et l'autre aboutissent à la même conclusion: le français perd aux Pays-Bas, durant le XVIII siècle, la place sinon « universelle » mais du moins privilégiée qui était la sienne à la fin du XVII ${ }^{\mathrm{e}}$ siècle, au profit non seulement du néerlandais mais aussi de l'allemand et de l'anglais. Leurs élites bilingues (néerlandais-français) s'y ouvrent au plurilinguisme européen ou non (en particulier, celui des Indes néerlandaises).

12 Ensuite, le couple formé par les contributions de Khavanova et de Horbec \& Matasovic, qui traitent l'une et l'autre du latin - plus exactement de ce qu'on appelait, dans les négociations diplomatiques, le «style de l'empire » cher aux Habsbourg de Vienne -, mais aussi des multiples vernaculaires (langues ou dialectes) pratiqués dans le vaste 
royaume de Hongrie, dont Ferdinand de Habsbourg avait hérité en 1526 à la mort de son beau-frère Louis II Jagellon.

Khavanova - études à l'université Lomonosov de Moscou poursuivies à la Central European University de Budapest - adopte un point de vue relativement extériorisé par rapport à son terrain d'étude, celui de la résistance des titulaires des divers offices impériaux à délaisser le latin, emblématique de leur profession, pour un allemand écrit que beaucoup d'entre eux ne maîtrisaient pas. Ces élites administratives furent bousculées par l'impératrice Marie Thérèse avec quelques ménagements, plus radicalement ensuite par son fils Joseph II, l'un et l'autre étant désireux de passer, en deux ou trois décennies, du quasi millénaire «style de l'empire » à l'allemand.

Horbec \& Matasovic - recherche financée par the Croatian Science Foundation - adoptent un point de vue que nous dirons, par euphémisme, moins distancié. En atteste le titre même de leur contribution (p. 111): Voices in a country divided, sous-titré Linguistic choices in early modern Croatia, ce qu'il nous faut, aussi brièvement qu'il nous est possible, commenter ${ }^{2}$.

15 Ce " pays divisé » est celui de la république de Croatie actuelle, dans les frontières qui sont les siennes depuis 1991, lesquelles l'auraient enfin réuni. C'est celui des «terres croates " (Croatian Lands) qui, au XVIII ${ }^{\mathrm{e}}$ siècle, relevaient d'au moins six souverainetés différentes : 1) un royaume de Croatie-Slavonie associé au royaume de Hongrie depuis le XII ${ }^{e}$ siècle et passé sous la tutelle des Habsbourg depuis le $\mathrm{XVI}^{\mathrm{e}}$ siècle (cf. ci-dessus); 2) la république de Venise ; 3) la république indépendante de Raguse (actuellement Dubrovnik) ; 4) l'empire ottoman qui occupait alors des «terres croates» en BosnieHerzégovine; 5) une vaste marche militaire (The Military Frontier ${ }^{3}$ ) administrée directement par Vienne ; 6) diverses « terres croates » qui relevaient du multiséculaire héritage Habsbourg. Il en résultait, selon un des sous-titres de cette contribution (p. 114), où il faut là aussi prêter attention aux pluriels et à l'unique singulier: "The confusion of tongues": official languages, languages of (high) society, and the mother tongue.

Dans ces « terres croates » de l'actuelle Croatie, il y avait au XVIII siècle presque autant de langues officielles que de souverainetés: le royaume de Croatie-Slavonie, tout comme celui de Hongrie, s'en tenait au "style de l'empire ", à ce néo-latin administratif et diplomatique emblématique du Saint empire romain germanique, latin qui s'y est maintenu, selon cette contribution (p. 115), « jusqu'en 1847 », date où cette monarchie devint la double monarchie (François-Joseph est empereur à Vienne et roi en Hongrie) ; toutefois, dans The Military Frontier et les terres héréditaires des Habsbourg (à une exception près), la «langue administrative» était l'allemand (p.116); les «terres croates " relevant de Venise usaient de l'italien, dont on peut se demander si un peu de vénitien n'y contaminait pas l'italien identitaire de l'Italie de la fin du XIX ${ }^{\mathrm{e}}$ siècle ; Raguse recourait soit à cet italien mâtiné de vénitien soit au néo-latin; et celles relevant encore de l'empire ottoman avait recours à l'osmanli de lointaine ascendance ouïgour (mais écrit en caractères arabes). Quant aux langues pratiquées par la (haute) société plus ou moins cosmopolite, elles incluaient, peu ou prou, ces langues officielles mais aussi le français, l'anglais, le hongrois, le roumain, voire le russe ou le turc.

Reste la langue maternelle, justification de l'existence et de l'étendue des «terres croates ». Elle est celle (p. 118 et sq.) de diverses «communautés rurales » dont une « vaste majorité » la parlait nativement - au moins dans l'une ou l'autre de ses variétés vernaculaires -, bref un ensemble de parlers paysans que "la langue littéraire croate " n'avait pas encore unifiés. On la dénommait certes Croatian mais aussi Slavonic ou 
Illyrian (p. 115) - par métonymie des «terres » où elle était nativement et majoritairement pratiquée -, bref un ensemble de parlers paysans, mutuellement plus ou moins intercompréhensibles, que les premiers philologues de la langue des croates regroupèrent en trois dialectes principaux (p. 123). Elle ne deviendra, dans la réalité socio-politique, la langue nationale (the national language, p. 118) de l'ensemble des «terres croates » qu'un demi-siècle plus tard, après que la double monarchie eut enfin accepté en 1847 que le hongrois en Hongrie et l'allemand dans le reste de l'empire finissent par détrôner définitivement le «style de l'empire ».

Quand Marie Thérèse d'Autriche écrivit à ses ministres, en 1773, qu'elle ne signerait plus rien écrit en latin (p. 93), quand son fils, un de ces «despotes éclairés » chers aux Lumières françaises, ordonna, en 1784, que l'allemand remplacerait le latin dans l'ensemble de ses services administratifs (p. 91), l'un et l'autre ne se doutaient pas qu'en ouvrant la boîte de Pandore des nationalités et des langues « nationales ", ils signaient, moins d'un siècle plus tard, la fin de la gloire des Habsbourg.

19 Une divergence entre la contribution de Khavanova et celle de Horbec \& Matasovic nous paraît, à cet égard, symptomatique de leur point de vue respectif : Khavanova estime que la Ratio educationis de Joseph II parvint rapidement à former une génération de lettrés aptes à remplacer, pour avoir étudié dans le secondaire «l'allemand, les mathématiques ou les statistiques » (p. 106), les générations plus âgées adeptes du néolatin administratif; Horbec \& Matasovic (p. 116) n'y voient, quant à elles, qu'un des échecs du réformisme « éclairé » de Joseph II (just one among many unccesseful reforms of Joseph II).

Les deux contributions qui terminent l'ouvrage sont celles de Rjéoutski et de Kislova.

De celle de Rjéoutski, nous faisons une lecture un peu différente de ce qui en est dit dans l'introduction de l'ouvrage (signée Rjéoutski \& Frijhoff): la noblesse russe du $\mathrm{XVIII}^{\mathrm{e}}$ siècle était certes capable de voir ce que le latin était à même d'apporter au prestige héréditaire qui était le sien, mais elle préféra miser sur le français, plus que sur l'allemand, pour le conforter (p. 10) ; elle en dédaigna donc ce latin que Pierre le Grand, afin de sortir l'église orthodoxe russe de son incurie intellectuelle, était parvenu à lui imposer, ce qui fit du français un « instrument de [1]a suprématie culturelle » (p. 11) de la noblesse.

Thèse que Rjéoutski a brillamment défendue dans ses écrits antérieurs, particulièrement dans ceux portant sur le corps des cadets de Saint-Pétersbourg aux $\mathrm{XVIII}^{\mathrm{e}}-\mathrm{XIX}{ }^{\mathrm{e}}$ siècles. Sa contribution au présent ouvrage ne dément pas cette thèse, mais la nuance quelque peu (certaines de ses notes sont à cet égard révélatrices). Pour ce qui est du latin - dont le titre de sa contribution dit que ce fut the history of a defeat -, le curriculum du corps des cadets de Saint-Pétersbourg n'était pas le même que celui des écoles dépendant de l'académie des sciences de Saint-Pétersbourg ou de l'université de Moscou : laissé au libre choix des cadets, il ne l'était pas chez les étudiants ( $c f . p p .171$ et sq.). L'origine sociale des uns et des autres jouait aussi son rôle (pp. 177-180). Des membres de la haute noblesse russe, hommes et femmes, ne dédaignaient pas le latin, l'associant à «la bonne éducation » (p. 179) et l'imposant à leurs enfants, y compris parfois à leurs filles (note 41). D'où une conclusion (pp. 183-185), où Rjéouski précise et infléchit celle de ses écrits antérieurs.

23 Une évolution qui doit beaucoup, nous semble-t-il, aux travaux d'Ekaterina Kislova qu'il remercie dans ses notes 3 et 24. La contribution de Kislova est une de celles qui m'ont le plus intéressé, peut-être en raison de ma quasi ignorance du domaine dont elle traite. 
Elle y rend compte savamment, à partir de trois fonds d'archives (un à Moscou, deux à Saint-Pétersbourg) et d'une bibliographie de plus de quatre pages, de la manière dont le pouvoir politique russe, celui de Pierre le Grand et de ses successeur(e)s, s'y est pris, au cours du XVIII siècle, pour faire du latin, langue de l'église de Rome depuis au moins la Vulgate, la langue non certes de l'église orthodoxe russe, mais celle permettant à une partie de son haut clergé d'accéder à une « éducation » dont le clergé cantonné au russe et au slavon d'église était dépourvu.

Une manière impériale qu'on peut grossièrement résumer ainsi. En 1710, Pierre le Grand dissocie visuellement (p. 195) ce slavon d'église mêlé de russe, qui était jusqu'alors la langue littéraire de son empire, d'un russe plus courant : il décrète que les livres religieux seront imprimés en caractères cyrilliques traditionnels (Church Slavonic letters) et les livres séculiers dans des caractères en partie différents (secular books in Russian - in civil fonts), façon aussi d'émanciper le russe de sa gangue religieuse. La tradition (pp. 201-202), d'ordre corporatif au sens médiéval, voulait que les popes assurent eux-mêmes la formation spirituelle et intellectuelle de leurs fils afin qu'ils puissent leur succéder dans leur ministère, ce qui rendait difficile le contrôle tant de leur nombre que de leur compétence intellectuelle. Pierre le Grand s'appuya sur une partie de son clergé plus ou moins familiarisé au latin - celui voisin des terres catholiques de Pologne et d'Autriche ou des terres luthériennes d'Allemagne et de la Baltique - pour susciter la création de séminaires publics où le latin était obligatoirement enseigné aux futurs popes, l'échec aux examens ayant pour sanction de les réduire à la condition de marin ou de paysan, deux formes de la même servitude.

Une latinisation dont le « despotisme éclairé » n'était déjà pas absent et qui, en deux ou trois générations, fit du latin une langue que Kislova qualifie de "professional" ou "corporate", c'est-à-dire à même de promouvoir un clergé orthodoxe "éduqué " capable de suivre et d'affronter les clergés catholique ou luthérien dans les controverses théologiques ou philosophiques européennes, un clergé dont le latin légitimait la supériorité hiérarchique par rapport au clergé «non éduqué », pour qui le latin était resté ce qu'il était séculairement dans l'orthodoxie russe, une langue « hétérodoxe ».

Restent deux contributions rétives à nos couplages.

Celle de Rocher en est toutefois proche, en ce qu'il y compare l'enseignement, au XVIII siècle, des langues anciennes (latin, grec et hébreu) et des langues étrangères (dont le français) dans quatre grammar schools de l'Allemagne centrale - entendue comme le " polygone » Berlin-Magdeburg-Dresden-Eisenach (p. 145, note 2) - et deux autres situées à Reval (Tallinn dans l'actuelle Estonie). Dans ces écoles réputées, que Rjéoutski \& Frijhoff qualifient à juste titre de Latin schools in Germany (p. 9), on enseignait le latin (moins le grec et peu l'hébreu) grammaticalement à la manière des jésuites dans leurs collèges mais aussi des langues vulgaires, natives ou non. Alors que, tant dans les universités du Saint empire romain germanique que de la Baltique estonienne, on n'entrait pas sans être quelque peu latinisé.

Les élèves (garçons) de ces six grammar schools étaient recrutés majoritairement dans les couches supérieures de la société mais pas exclusivement, les quatre allemandes étant situées dans une région très majoritairement germanophone, et les deux baltiques dans une région multilingue (allemand, suédois, russe, estonien et autres parlers ethniques). Mais les unes et les autres étaient fréquentées majoritairement par des élèves germanophones ${ }^{5}$, et Rocher adopte la même méthode, plus sociologique que 
linguistique ou didactique, pour les comparer. On y perd donc un peu de ces divergences de point de vue qui font l'intérêt de nos couplages.

Rocher postule que deux motivations polaires sont à même de déterminer le choix des langues par ces élèves issus de milieux privilégiés : une utilitaire, liée à leur future carrière ; une qu'on pourrait dire identitaire, liée au désir d'être conforme à l'entre-soi distinctif de la classe supérieure dont ils provenaient ou dont ils aspiraient à faire partie. Sa conclusion est que, à la fin du XVIII ${ }^{e}$ siècle, les élèves des quatre écoles allemandes délaissent le latin au profit de l'allemand et des langues étrangères (français, italien, anglais) à des fins plutôt d'ordre utilitaire (p. 155), alors que ceux des deux écoles baltiques continuent à privilégier le latin à des fins plutôt de distinction sociale, tout en ne dédaignant pas le français ou le russe aux mêmes fins ou d'autres.

La contribution de Bruschi, qui ouvre ce volume, est intitulée Learning Vernaculars, Learning in Vernaculars, ce que son résumé explicite comme l'apprentissage des langues vulgaires en les employant comme outil pour enseigner des disciplines non prises en compte dans les collèges et les universités (p. 15). Une approche qu'il apparente (p. 30, note 36) à ce qu'on dénomme de nos jours Content and Language Integrated Learning (ou CLIC) $)^{6}$

31 La singularité de la contribution de Bruschi est, à nos yeux, qu'elle est la seule de ce volume à traiter du français en France entre la fin du XVI ${ }^{e}$ siècle et le milieu du XVIII ${ }^{e}$ siècle, et elle en traite à propos de la formation tant physique qu'intellectuelle des enfants de la noblesse. Il y est question du plan d'études du huguenot François de la Noue dans le cinquième de ses « discours politiques et militaires » (1583), qui proposait d'ouvrir en France huit "académies» dont le français serait la seule langue d'enseignement, les textes anciens y étant traduits en français. On y enseignerait dans cette langue, maternelle ou non des élèves, les « exercices » chers à la noblesse d'épée (équitation, danse, escrime, natation, lutte, art des fortifications), mais aussi la géographie, l'histoire moderne ou ancienne, la philosophie morale et la stratégie militaire, des savoirs négligés par les collèges et les universités (p. 20). Il y est aussi question de l'éphémère école (1640-1646) fondée par Nicolas le Gras dans la ville de Richelieu (celle du cardinal), dont le programme incluait aussi les « exercices » propres à la noblesse, mais aussi les «Arts de la Paix \& de la Guerre » à même d'assurer les «perfections de l'Esprit» (p. 21, note 21).

Deux projets d'une formation spécifique à la jeunesse nobiliaire française qui, selon Bruschi, inspireront à la fin du XVII ${ }^{e}$ siècle et au XVIII ${ }^{e}$ siècle, nombre de manuels de "grammaires " bilingues ou non (p. 24 et sq.). Il y a là matière à intéresser les lecteurs de Documents : ni la Noue ni le Gras n'y sont à ma connaissance apparus, et s'il y est question d'une partie des "grammaires » citées par Bruschi, elles y sont étudiées sous un autre angle que le sien, celui de l'actuel et néanmoins séculaire CLIC. Il y a aussi matière à encourager les membres de la SIHFLES à travailler sur un domaine jusqu'alors peu exploré, celui du rôle des écoles militaires dans la promotion des langues vulgaires en tant que langues d'enseignement, au moins un siècle avant qu'on ne commence à en faire autant dans les collèges et universités.

On aura compris que, dans cet ouvrage, il s'agit plus de l'Europe telle que l'entendait le général de Gaulle, allant « de l'Atlantique à l'Oural », que de celle de Bruxelles.

Nombre de notions auxquelles recourent ses contributeur(e)s posent question à qui est un peu attentif aux outils conceptuels utilisés pour raisonner du choix des langues dans un passé structuré socialement, culturellement, religieusement et politiquement d'une 
autre façon que notre actuelle Europe. On peut certes parler de l'aristocratie au XVII ou au XVIII siècle, mais est-ce ainsi que les noblesses d'Europe se qualifiaient alors? Les ordres d'Ancien régime s'énumèrent généralement en français ainsi : la noblesse y précède le clergé, lesquels précèdent le tiers état. En allait-il toujours de même dans le Saint-empire romain germanique ou dans la Russie d'avant Pierre le Grand où parfois le clergé était, semble-t-il, placé avant la noblesse ? À ces risques d'anachronismes mineurs s'ajoutent des questions plus générales. Dans l'Europe des $\mathrm{XVII}^{\mathrm{e}}-\mathrm{XVIII}{ }^{\mathrm{e}}$ siècles, il n'existait pas d'État dont les frontières coïncidaient avec l'aire d'usage majoritaire d'une langue ${ }^{7}$, et les souverains se glorifiaient parfois de la multiplicité des langues parlées par leurs sujets. Mai en quoi l'empire des Habsbourg de Vienne était-il plus multilingue ou multiethnique que le royaume des Bourbon de Paris? Le «principe des nationalités » tel qu'entendu aux XIX ${ }^{\mathrm{e}}$-XXe siècles est-il applicable, sans anachronisme, aux deux siècles précédents?

Il reste que le choix des langues, tel qu'il est traité dans cet ouvrage, concerne des territoires, des langues et des pratiques socioculturelles qui n'ont été jusqu'alors peu étudiés, au moins en français. Le soin et la compétence avec lesquels cet ouvrage a été édité permet de s'en instruire en anglais, y compris pour qui n'en est pas natif. Autant de raisons pour en recommander la lecture.

\section{NOTES}

1. On trouvera dans le dernier numéro de La Lettre de la SIHFLES ( ${ }^{\circ} 79-80$ octobre 2018, p. 45) la Table of Contents de cet ouvrage, ainsi que sa quatrième de couverture et son prix «\$105».

2. Cf. la carte des Croatian Lands in the 18th century p. 115. Sans doute en couleurs à l'origine, le noir et blanc de sa reproduction en confond les différents gris, la rendant difficilement lisible.

3. Les historiens français parlent des confins militaires. Les auteures ne disent pas que, dans ces " confins », on trouvait certes des croatophones indigènes mais aussi des croatophones qui ne l'étaient pas (en particulier ceux engagés par l'armée autrichienne), ainsi que des orthodoxes serbes qui parlaient des dialectes plus ou moins intercompréhensibles avec certains des dialectes croates.

4. Les guillemets sont ceux de Kislova.

5. Les chevaliers teutoniques s'étaient emparés de Reval à la fin du XIV e siècle, et les germanophones y tenaient depuis lors le haut du pavé.

6. L'équivalent français de ce sigle est EMILE: Enseignement d'une matière par intégration d'une langue étrangère. Pour ce qui concerne le secondaire, on parle d'enseignement en classe bilingue ou section bilingue avec à peu près le même sens : « enseigner en langue étrangère ou seconde une autre matière qu'elle-même ».

7. En va-t-il différemment de nos jours? 


\section{AUTEUR}

HENRI BESSE

ENS de Lyon 